\title{
ActiThings: Motivating the Prevention of Frailty
}

\author{
Jochen Meyer \\ OFFIS - Institute for Information \\ Technology \\ Germany \\ jochen.meyer@offis.de
}

\author{
Elke Beck \\ OFFIS - Institute for Information \\ Technology \\ Germany \\ elke.beck@offis.de \\ Susanne Boll \\ University of Oldenburg \\ Germany \\ susanne.boll@uni-oldenburg.de
}

\author{
Kai von Holdt \\ OFFIS - Institute for Information \\ Technology \\ Germany \\ kai.von.holdt@offis.de
}

\begin{abstract}
Simple home-based physical activities are effective in the prevention of frailty for elderly persons, but adherence to such programs is challenging. We suggest the ActiThings toolkit for the provision of environmental prompts in opportune moments. We describe the design of two functional prototypes to motivate for exercising in opportune moments and to tackle sedentary behavior. We outline the evaluation results and discuss implications for future work.
\end{abstract}

\section{CCS CONCEPTS}

- Human-centered computing $\rightarrow$ Empirical studies in ubiquitous and mobile computing

\section{KEYWORDS}

Motivation technology, opportune moments, prevention, frailty, elderly persons

\section{INTRODUCTION}

Lifelong physical activity is one of the key determinants of healthy aging. However, globally, 1 out of 4 adults is insufficiently active. This is a particular challenge in higher age, where lack of physical activity contributes to frailty, leading to

*Produces the permission block, and copyright information †The full version of the author's guide is available as acmart.pdf document ${ }^{1}$ It is a datatype. falls, injuries, and a loss of independence. Older adults are therefore recommended to perform exercises for maintaining endurance, strengthening the muscles, and enhancing balance.

Exercise programs such as HOPE [2] address these needs und suggest activities, that can be conducted at home with relatively low efforts, and that are proven effective. Still, insufficient longterm adherence to such exercise programs is a reoccurring problem. Rather than advocating structured exercise programs, it is a promising approach to conduct exercises during daily life activities, in an unobtrusive manner, and with little extra effort. This requires changes of behaviors and habits, which is a considerable challenge. But it has been shown that supervision and motivational support of the participating people provide encouragement to exercise and thus contribute to a better program adherence [1].

Our goal is therefore to develop technologies that increase adherence of elderly persons to home-based physical exercise programs integrated in daily lives by reminding about exercises in opportune moments.

\section{APPROACH}

We deploy the successful concept of environmental prompts [4] to cue older adults about performing physical exercises. We suggest the ActiThings toolkit, a modular toolkit comprising high- and low-fidelity, small, easy to use, ambient technologies. We use a set of different sensors for detecting opportune moments, i.e. points in time where a person has the capability and willingness to be reminded about physical exercise and to conduct it. We aim to detect routine activities that may fulfil the conditions for performing a short physical exercise. We hypothesize that such moments may be before - e.g. while approaching the coffee machine -, during - e.g. while waiting for the water to boil -, or after - e.g. after watering the flowers - a routine household activity. Due to the broad range of potential individual opportune moments, we envision a set of different sensors that can flexibly be used in different contexts.

Next to detecting opportune moments, suitable interactive technology needs to notify an older adult about this physical exercise opportunity. In order to be effective, reminder systems need to be highly personalizable, enabling the person to choose between diverse modalities such as vision, speech, sound, and touch [3]. We again envision a range of different components 
providing e.g. acoustic and visual environmental prompts to do a simple physical exercise.

We subsequently describe the design and evaluation of first prototypes (see Fig. 1) of such components for detection of opportune moments and for reminding and motivating physical activity.
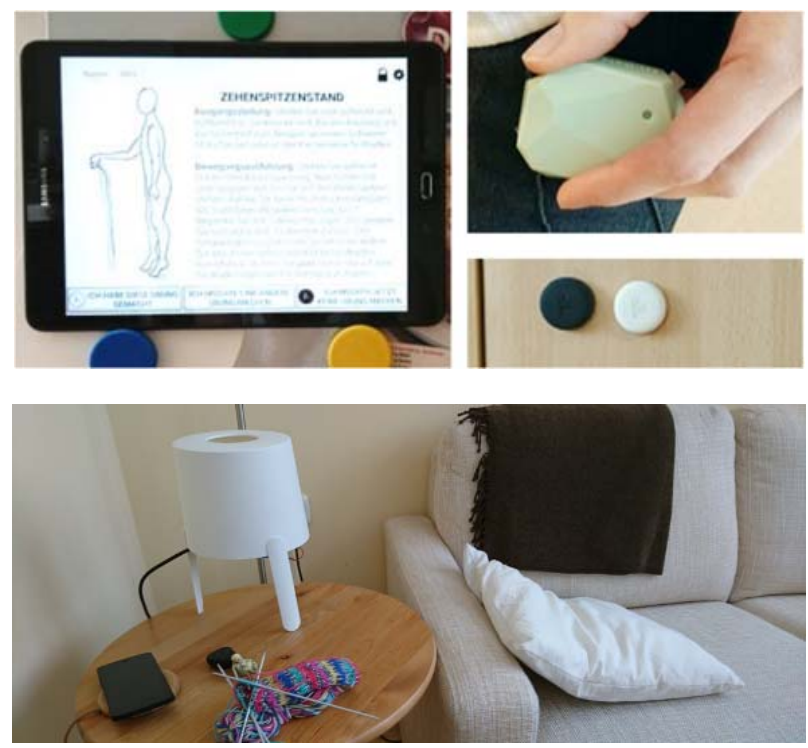

Figure 1: top: ActiConverge with tablet PC, Bluetooth beacon, and Bluetooth buttons. bottom: ActiSit with table lamp and pillow.

\section{DESIGN PROCESS AND PROTOTYPES}

ActiConverge identifies a person entering a room. Users are equiped with near-body-worn Bluetooth beacons. A tablet PC, which can e.g. be mounted to a cupboard or placed on a flat surface, recognizes, when a user with a beacon comes close and suggests a random simple physical exercises such as toe-heelsstand. Additionally the tablet provides an image of a person performing the exercises and a short explanation of how to execute the exercise. Having either performed an exercise, or preferring not to do one right now can be acknowledged by the press of a physical Bluetooth or an on-screen button. In case the suggested exercise doesn't fit the users situation or preferences, he can also choose a different one or have another one randomly chosen.

The ActiSit prototype collects sensor data on sitting to tackle sedentary behavior. A pillow is equipped with a force sensitive resistor that identifies a sitting person and measures the time of sitting. A table lamp serves as an ambient light display to motivate physical activity. After the maximum of predefined continuous (i.e. uninterrupted sitting without standing up) or cumulative (i.e. total time spent sitting during a day) sitting time is reached, the lamp changes its color to notify the user. The first notification from neutral white to yellow happens after one hour of continuous or six hours of cumulative sitting time. The second notification takes places after two hours of continuous or seven hours of cumulative sitting time by changing the lamp light from yellow to red.

We evaluated the prototypes with five older adults ( 2 female, aged 71-80) in an apartment-like lab providing a close-to-real-life environment. After a pre-interview, the participants tried out the prototypes and verbalized their experiences and first impressions. A semi-structured interview aiming at experiences and perceptions concluded the evaluation.

The participants experienced the prototypes as useful. They pointed to important restrictions, such as not being reminded when visitors are around or during an after-lunch nap, and recommended extensions such as an acoustic modality for notifications. They suggested that existing personal devices that they already use should be integrated into the system, such as smartphones, tables, or e-readers.

\section{DISCUSSION AND OUTLOOK}

Our study indicates that technology in daily lives may contribute to an improved adherence to home-based physical exercise programs. The evaluation confirms the importance of tailoring the reminders and the sensing and feedback technologies to the individual needs and preferences of older adults. The devices of the ActiThings toolkit need to be able to sense behavior and provide reminders at many different locations at home. Thereby, particular sensing and notification devices seem to fit particular situations at home. Accustomed, personal smart devices such as smartphones or e-readers should be integrated for tracking their time of use and for providing prompts to do a physical exercise. Moreover, tablet PCs work well as ambient reminder displays, particularly when placed in highly frequented places at home.

Based on our research findings so far, we see a high potential for our approach to integrate physical activity in daily life. The ActiThings toolkit prototypes enable environmental prompts to physical exercise in a variety of situations at home, taking individual needs and preferences of older adults into account. In future work we aim to build a platform facilitating the flexible integration and configuration of different sets of sensors and actors, tailored to the user's individual needs.

\section{ACKNOWLEDGMENTS}

This research was funded by the German Federal Ministry of Education and Research (AEQUIPA, Project No. 01EL1422D).

\section{REFERENCES}

[1] Dinnie Chao, Capri G Foy, and Deborah Farmer. 2000. Exercise adherence among older adults: challenges and strategies. Contemporary Clinical Trials 21, 5: S212-S217.

[2] Andrew Clegg, Sally Barber, John Young, Steve Iliffe, and Anne Forster. 2014. The Home-based Older People's Exercise (HOPE) trial: a pilot randomised controlled trial of a home-based exercise intervention for older people with frailty. Age and ageing 43, 5: 687-695.

[3] Marilyn Rose McGee-Lennon, Maria Klara Wolters, and Stephen Brewster. 2011. User-centred multimodal reminders for assistive living. In Proceedings of the SIGCHI Conference on Human Factors in Computing Systems, 2105-2114.

[4] Susan Michie, Stefanie Ashford, Falko F Sniehotta, Stephan U Dombrowski, Alex Bishop, and David P French. 2011. A refined taxonomy of behaviour change techniques to help people change their physical activity and healthy eating behaviours: the CALO-RE taxonomy. Psychology \& health 26, 11: 14791498. 